Review

\title{
Prognostic and Clinicopathological Value of Racl in Cancer Survival: Evidence from a Meta-Analysis
}

\author{
Shu Lou ${ }^{2}$, Penglai Wang ${ }^{1 *}$, Jianrong Yang 2 , Junqing Ma², Chao Liu ${ }^{1}$, Meng Zhou ${ }^{1,2}$ \\ 1. Department of oral and maxillofacial surgery, Xuzhou Stomatological Hospital, 130 Huaihai Road, Xuzhou, Jiangsu 221002, China \\ 2. Jiangsu Key Laboratory of Oral Diseases, Nanjing Medical University, 136 Hanzhong Road, Nanjing, Jiangsu 210029, China \\ *Shu Lou and Penglai Wang have contributed equally to this work. \\ $\square$ Corresponding authors: Meng Zhou (E-mail: drzhoumeng87@126.com) or Chao Liu (E-mail:liuchao2299@163.com) \\ (c) Ivyspring International Publisher. This is an open access article distributed under the terms of the Creative Commons Attribution (CC BY-NC) license \\ (https://creativecommons.org/licenses/by-nc/4.0/). See http://ivyspring.com/terms for full terms and conditions.
}

Received: 2018.01.08; Accepted: 2018.04.15; Published: 2018.06.23

\begin{abstract}
Purpose: The role of Racl in cancer survival has been widely studied. However, the prognostic and clinicopathological value of Racl remains inconclusive. We performed a meta-analysis to clarify the role of Racl in cancer survival as well as its association with clinicopathological features.

Methods: Eligible studies were searched from PubMed, Cochrane Library, Embase, and Web of Science databases. The pooled hazard ratios (HRs) and odds ratios (ORs) with corresponding $95 \%$ confidence intervals (Cls) were used to detect the prognostic and clinicopathological role of Racl.

Results: A total of 14 studies including 1793 patients were enrolled in the present meta-analysis. Pooled HR for overall survival (OS) (HR=2.02, 95\% Cl: 1.70-2.39) and disease-free survival (DFS) $(\mathrm{HR}=2.64,95 \% \mathrm{Cl}$ : 1.71-4.09) indicated a significant poor prognostic effect for Racl. Positive Racl expression was found to be correlated with tumor stage, blood vessel invasion, and lymph metastasis, but not with histological differentiation. Sensitivity test showed no single study altered OS or DFS significantly. No publication bias was detected by Egger's test and Begg's funnel plot test.

Conclusion: This meta-analysis indicated that Racl could be used as a potential marker to predict cancer prognosis. Additionally, Racl expression was associated with the malignancy-related phenotype.
\end{abstract}

Key words: Rac1, cancer, prognosis, biomarker, Meta-analysis

\section{Introduction}

Cancer is a leading cause of death in the world. It is estimated that 14.1 million new cancer cases and 8.2 million cancer deaths occur annually in the world, and this number is still on the rise [1]. Although great progress has been made in the clinical diagnosis and treatment, the prognosis of cancer patients is still unsatisfactory. Many cancer patients are diagnosed in a late stage, leading to a poor postoperative survival [2-4]. Thus, a reliable and validated biomarker reflecting the precise biological behavior of tumor would be valuable for clinical decision-making and the improvement of the curative effect [5].

Rho GTPases are a subfamily of the small G proteins with 20 members and have been recently considered as cancer-related biomarkers [6]. Rho GTPases were reported to participate in the regulation of cell polarity, proliferation, migration, and angiogenesis in diverse cancer cells $[7,8]$. Ras-related C3 botulinum toxin substrate 1 (Rac1), a member of the Rho GTPase family, has been recognized as a key marker of tumor invasion and metastasis [9]. The main functions of Rac1 signaling are regulating actin cytoskeleton by activating p21-activated kinases (PAK1, PAK2, and PAK3), promoting cell proliferation through the mitogen-activated protein kinase (MAPK) system, and modulating the inflammatory response via NF-kB pathway [10-12]. Rac1 also plays a crucial role in ROS-mediating cell 
killing and G1 cell-cycle progression [13]. These biological and pathological functions of Rac1 appear indispensable to tumor initiation, angiogenesis, invasion, and metastasis. Thus, the potential role of Rac1 in tumor development has drawn extensive attention.

Tumor angiogenesis is necessary for tumor nutrition and growth, and a major physiological function of Rac1 is vascular morphogenesis [6]. Blockade of Rac1 in endothelial cells resulted in the failure of lumens formation and endothelial cell invasion in three-dimensional collagen matrices [14]. Rac1 deletion in endothelial cells using a mouse model resulted in hemorrhage and edema due to a failure of lymphatic cell separated from vessels and lymphatic structures formation [15]. A recent study showed that gastric cancer tissue with positive Rac1 expression had a higher microvessel density than that with negative expression, suggesting that Rac1 promoted the formation of cancer blood vessels and the growth of tumor [16].

Recent studies have emphasized an integral role of Rac1 in tumor progression and metastasis. Rac1 modulates actin cytoskeleton recombination and determines the epithelial-to-mesenchymal transition (EMT) pattern, which is warranted for the acquisition of an invasive phenotype [17]. Silencing Rac1 in human melanoma cells led to a weaken pattern of cell proliferation and invasion, while overexpression of Rac1 in mouse keratinocytes induced EMT and promoted cell migration and invasion $[18,19]$. Studies also showed that high expression of Rac1 was correlated with local invasion and lymph node metastasis in upper urinary tract cancer [9]. Since tumor growth and metastasis have strong effects on the morbidity and mortality of cancer patients, the interplay between Rac1 and tumor metastasis suggests that Rac1 may be correlated with cancer prognosis.

To date, elevated expressions of Rac1 have been detected in various types of cancer (gastric cancer, breast cancer, esophageal cancer, lung cancer, gallbladder cancer, hepatocellular cancer, ovarian cancer et al) $[9,16,17,20-35]$. It has been demonstrated that overexpression of Rac1 was linked to aggressive growth and other malignant characteristics of tumor. High level of Rac1 could predict a poor prognosis in different types of cancer. However, a study conducted in Germany found that there was no association between Rac1 expression and prognosis of gastric cancer [21]. These conclusions are inconsistent and controversial. Thus, we performed a meta-analysis to summarize the existing data and demonstrated the prognostic and clinicopathological value of Rac1 in cancer patients.

\section{Materials and Methods}

\section{Search strategy}

This meta-analysis was conducted by the reporting checklist, and details of the list are available in Table S1. A bibliographic search for eligible literature updated to December 10, 2017 from the PubMed, Cochrane Library Web of Science, and Embase database was conducted by two investigators (Lou and Liu) independently. The following keywords were used in the search process: ("Rac1" or "Ras-related C3 botulinum toxin substrate 1" or "rac-1") and ("cancer" or "carcinoma" or "tumor" or "neoplasm") and ("survival" or "prognosis" or "prognostic" or "outcome"). Manual search for additional eligible studies in the reference lists was also performed.

\section{Inclusion and exclusion criteria}

All studies should meet the following criteria: (1) cohort or case-control study; (2) assessing the prognostic role of Rac1 in cancer; (3) conforming cancer diagnosis by pathological methods; (4) providing sufficient information for calculating hazard ratio (HR) with 95\% confidence interval (CI); (5) published in English. Articles were excluded if they met any item of the following criteria: (1) comment letters, case report or conference paper; (2) without available data to calculate the HRs and 95\% CIs; (3) in vitro and experimental studies, clinical cross-sectional studies; (4) studies not focused on the independent role of Rac1 in cancer survival. The included studies should meet the above standards. Two reviewers (Lou and Liu) identified the eligible studies independently according to the inclusion and exclusion criteria. Disagreement between the two reviewers was resolved by discussion with another reviewer (Zhou). The workflow of the literature identification is available in Figure 1.

\section{Data extraction and quality assessment}

Two reviewers (Wang and Yang) performed the data extraction independently using the same procedures and disagreements were resolved when a third reviewer (Ma) intervened. The following data were extracted: the first author, year of publication, geographic region, type of cancer, the information of preoperative treatment, detection method of Rac1, cut-off value, follow-up period, expression of Rac1, age, tumor stage/grade, clinical outcomes, multivariate/univariate analysis model and clinicopathological characteristics. When the HRs with corresponding 95\% CIs were provided in the articles directly, we used the crude ones. If the prognostic results were only shown as Kaplan-Meier curve, we 
extracted the data using the software Engauge Digitizer version 9.0. The quality of included studies was evaluated by two reviewers (Ma and Zhou) independently according to the NewcastleOttawa Scale (NOS) criteria [36]. The range of quality scores is 0 (lowest) to 9 (highest), and studies with a score more than 6 were marked as high quality. An independent investigator (Yang) made the final decision if there were disagreements between the initial two reviewers.

\section{Statistical Analysis}

In this meta-analysis, the pooled hazard ratios (HRs) with their 95\% confidence intervals (CIs) were used to evaluate the association between Rac1 expression and prognosis of cancer patients, and an HR >1 represented poor prognostic outcome for patients with elevated Rac1 expression. We also calculated the pooled odds ratios (ORs) with corresponding 95\% CIs to assess the correlation between Rac1 expression and clinicopathological features (tumor stage/grade, blood vessel invasion, lymph metastasis, and differentiation).

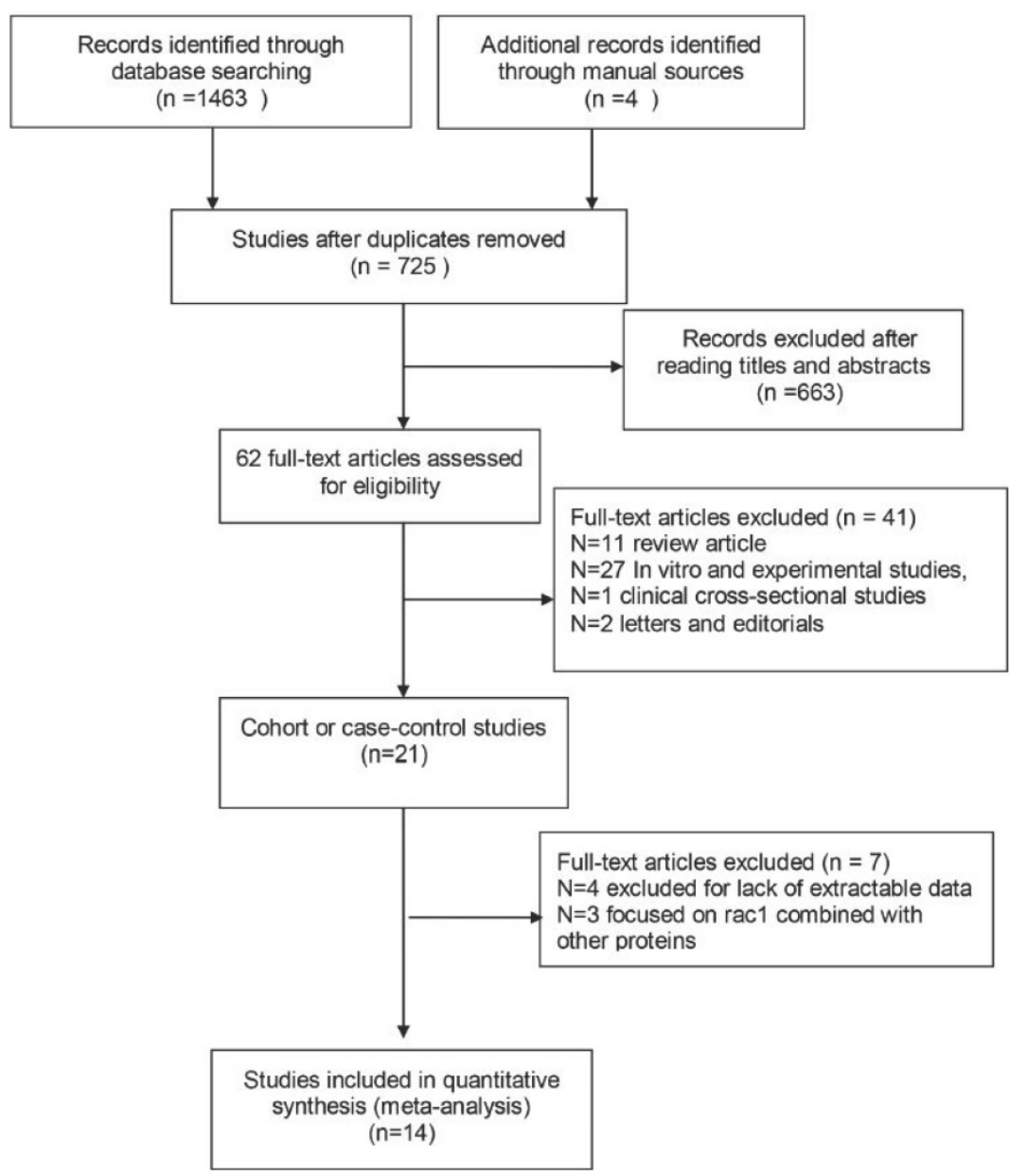

Fig 1. Flow diagram for literature selection process. The figure clarify how finally the identified studies were chosen from primary search records.
The statistical heterogeneity was determined by Chi-squared test and $I^{2}$ method. When the $P$ value for Chi-squared test (marked as $P_{\mathrm{H}}$ ) was less than 0.10 or $I^{2}$ was more than $50 \%$, suggesting the presence of heterogeneity, a random-effects model was used; otherwise, a fixed effect model was used. $Z$ test was used to examine the fixed/random effect model and $P$ value for $Z$ test $<0.05$ indicated a statistical significance. The potential publication bias was evaluated by Begg's funnel plots and further examined with the Egger's linear regression test; $P$ value for Egger's test or Begger's funnel plot $<0.05$ was considered as statistically significant. Sensitivity and subgroup analyses were performed to test the source of heterogeneity and stability of results. All statistical analyses were performed using STATA software version 12.0 (Stata Corporation, College Station, Texas, USA).

\section{Results}

\section{Literature search information}

A total of 1467 studies were retrieved from the databases according to the search strategy. The flowchart of the literature retrieved process is shown in Fig 1. In brief, after the initial screening based on the titles or abstracts and excluded duplicates, 1405 studies were excluded, leaving 62 studies for the full-text review. By further reviewing the remaining results, a total of 21 studies were left for further evaluation. Then 7 studies were excluded due to unavailable data for HRs with $95 \%$ CIs $(n=4)$ calculation and not focused on the independent role of Rac1 in cancer survival ( $n=3)$. Finally, 14 studies regarding the associations between Rac1 expression and cancer prognosis were included in our meta-analysis.

\section{Characteristics of included studies}

The characteristics of the eligible studies are available in Table 1. A total of 1793 (ranging from 60-242 for each study) patients in 14 studies were ultimately included in this metaanalysis. The publication year ranged from 2007 to 2017. Twelve studies were performed in Asia (11 in China and 1 in Japan), the other 2 studies were carried out in Germany. As for cancer type, four studies were about gastric cancer, two were about non-small cell lung cancer, one about prostate cancer, one about 
nasopharyngeal cancer, one about esophageal cancer, one about hepatocellular cancer, one about breast cancer, one about ovarian cancer, one about upper tract cancer, and one about gallbladder cancer. Regarding detection method, the expression level of Rac1 was evaluated by immunohistochemistry (IHC) in 13 studies, and western blotting (WB) was used in the remaining study. The cut-off value of IHC for Rac1 expression was based on the percentage of Rac1 positive cells. Rac1 positive expression rate was ranged from $36 \%$ to $86.78 \%$ in studies using IHC, and a positive expression rate of $42.59 \%$ was reported in the literature using WB detection method. Study endpoints were determined as overall survival (OS, 12 studies in total) and disease-free survival (DFS, 6 studies involved). All of the included studies were of high quality with scores of NOS $\geqq 6$.

\section{Prognostic value of Racl for OS and DFS}

Among the included studies, a total of 12 studies with 1571 cancer patients reported HRs for OS. As showed in Figure. 2a, no significant heterogeneity was detected in these studies, therefore the pooled HR (2.02, 95\% CI: 1.70-2.39, $\mathrm{I}^{2}=18.2 \%, P_{\mathrm{H}}=0.265 ; P_{\mathrm{z}}<$ $0.001)$ was calculated by the fixed effects model. As to Rac1 expression for DFS analysis, a total of 6 studies including 815 patients were enrolled, of which four studies directly provided multivariate HRs and two studies indirectly with univariate HRs. A poor prognostic effect of Rac1 for DFS of cancer patients was also found using the random effect model (pooled HR=2.64, 95\% CI: 1.74-4.09, $\mathrm{I}^{2}=60.4 \%, P_{\mathrm{H}}=$ 0.027; $P_{Z}<0.001 ;$ Fig. 2b).

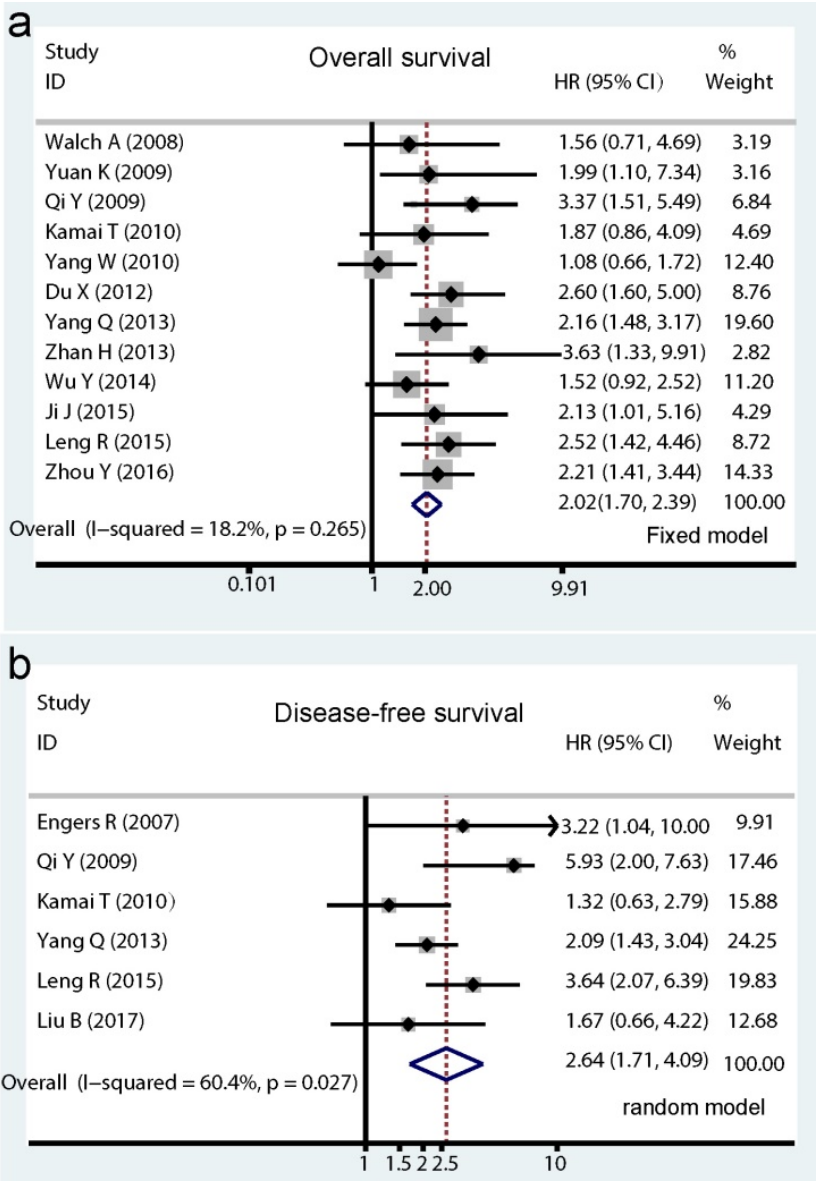

Fig 2. Forrest plots of pooled hazard ratios estimate for Racl impact on overall survival (OS) and disease-free survival (DFS). Results are presented as individual hazard ratio (HR), and $95 \%$ confidence interval $(\mathrm{Cl})$. The middle point of the diamond represents the pooled HR and its left and right corners represent $95 \% \mathrm{Cl}$. (a) shows pooled hazard ratio (HR) for overall survival (OS) analysis; (b) shows pooled HR for DFS analysis.

Table 1. Main characteristics of the studies included in the meta-analysis

\begin{tabular}{|c|c|c|c|c|c|c|c|c|c|c|c|c|}
\hline $\begin{array}{l}\text { First author } \\
\text { (publish year) }\end{array}$ & Country & Cancer type & PT & $\begin{array}{l}\text { Sample } \\
\text { size }\end{array}$ & $\begin{array}{l}\text { Detection } \\
\text { method }\end{array}$ & Cut off & $\begin{array}{l}\text { Follow-up time } \\
\text { (median/range) }\end{array}$ & $\begin{array}{l}\text { Rac1 } \\
\text { expression } \\
(\%)\end{array}$ & $\begin{array}{l}\text { Age } \\
\text { (median/ } \\
\text { range) }\end{array}$ & $\begin{array}{l}\text { Stage/ } \\
\text { grade }\end{array}$ & $\begin{array}{l}\text { Outcome } \\
\text { s, mode }\end{array}$ & $\begin{array}{l}\text { Quality } \\
\text { score }\end{array}$ \\
\hline Engers R (2007) & Germany & prostate cancer & no & 60 & IHC & $10 \%$ & $86(4-173)$ & 56.67 & $67(55-79)$ & II-III & DFS,M & 8 \\
\hline Walch A (2008) & Germany & gastric cancer & no & 76 & IHC & $10 \%$ & $38(1-120)$ & 67 & $69(30-86)$ & I-III & OS,U & 7 \\
\hline Yuan K (2009) & China & NSCLC & no & 111 & IHC & $10 \%$ & $33(2-47)$ & 36 & $60(26-79)$ & I-IV & OS,M & 7 \\
\hline Qi Y (2009) & China & NPC & no & 102 & IHC & $0 \%$ & $60(12-87)$ & 84.31 & $20-84$ & II-IV & $\begin{array}{l}\text { OS,M / } \\
\text { DFS,M }\end{array}$ & 8 \\
\hline Kamai T (2010) & Japan & $\begin{array}{l}\text { Upper tract } \\
\text { cancer }\end{array}$ & no & 108 & WB & $>2.72$ & $41(5-132)$ & 42.59 & $71.9(42-89)$ & I-III & $\begin{array}{l}\text { OS,M / } \\
\text { DFS,M }\end{array}$ & 8 \\
\hline Yang W (2010) & China & $\mathrm{HPC}$ & NA & 242 & IHC & $33 \%$ & 53 & 86.78 & 65.2 & I-IV & OS,U & 7 \\
\hline Du X (2012) & China & gallbladder & no & 86 & IHC & $10 \%$ & $36(12-66)$ & 79.1 & NA & I-II & OS.M & 6 \\
\hline Yang Q (2013) & China & EPC & no & 233 & IHC & $10 \%$ & $39.8(3-84)$ & 48.06 & NA & I-III & $\begin{array}{l}\text { OS,U / } \\
\text { DFS,U }\end{array}$ & 7 \\
\hline Zhan H (2013) & China & gastric cancer & no & 60 & IHC & $50 \%$ & $3-60$ & 61.67 & $56.5(35-78)$ & I-IV & OS,U & 8 \\
\hline Wu Y (2014) & China & gastric cancer & no & 158 & IHC & $50 \%$ & $26(1-60)$ & 67.72 & $56.25(28-83)$ & I-IV & OS,U & 7 \\
\hline Ji J (2015) & China & gastric cancer & NA & 92 & IHC & $5 \%$ & $39(1-80)$ & 72.8 & $63(37-84)$ & I-IV & OS,U & 6 \\
\hline Leng R (2015) & China & ovarian cancer & no & 150 & IHC & $0 \%$ & $45(1-120)$ & 55.33 & $52.3(17-89)$ & I-IV & $\begin{array}{l}\text { OS,M / } \\
\text { DFS,U }\end{array}$ & 8 \\
\hline Zhou Y (2016) & China & NSCLC & no & 153 & IHC & $50 \%$ & $57(4-95)$ & 72.55 & NA & I-IV & $\begin{array}{l}\text { OS,M / } \\
\text { PFS,U }\end{array}$ & 7 \\
\hline Liu B (2017) & China & breast cancer & NA & 162 & IHC & $33 \%$ & $79(1-122)$ & 59.88 & $53(28-84)$ & I-IV & DFS,M & 7 \\
\hline
\end{tabular}




\section{Subgroup analysis}

Pooled HRs and heterogeneity test results for different subgroups in OS and DFS analysis are available in Table 2. Although there was no inter-study heterogeneity in the quantitative synthesis of OS, subgroup analyses were performed on the basis of age, country of origin study, cancer type, cut-off value, follow-up time, sample size, multivariate/ univariate analysis, and preoperative treatment. There were significant associations between high Rac1 expression and OS in subgroups including age, country of origin study, cancer type, cut-off value, follow-up time, sample size, multivariate/univariate analysis. Only the subgroup without applicable preoperative treatment information revealed no significant correlation of positive Rac1 expression with OS (HR=1.29, 95\% CI: 0.85-1.94, $I^{2}=49.6 \%, P_{\mathrm{H}}=$ $\left.0.159 ; P_{\mathrm{z}}=0.233\right)$. With regard to Rac1 expression for DFS, only subgroups of age $\geqq 60 \mathrm{y}$ and patients without available preoperative information demonstrated no significant association between elevated Rac1 expression and DFS, other subgroup analysis demonstrated increased Rac1 expression was significantly linked to DFS. Detailed information of subgroup analysis is shown in Table 2.

\section{Correlation of Racl with clinicopathological features of cancer patients}

A total of 13 studies assessed the relationship between Rac1 expression and clinicopathological characteristics. Results showed that increased Rac1 expression was significantly associated with lymph metastasis $\left(\mathrm{OR}=2.64,95 \%\right.$ CI: $1.53-4.56, I^{2}=76.4 \%, P_{\mathrm{H}}=$ $0.000 ; \mathrm{Pz}<0.001 ;$ Fig. 3a), tumor stage/grade (OR=3.17, 95\% CI: 2.02-4.97, $I^{2}=69.7 \%, P_{\mathrm{H}}=0.000 ; P_{\mathrm{z}}<$ 0.001; Fig. 3b), and blood vessel invasion (OR=1.79, $95 \%$ CI: 1.12-2.85, $I^{2}=33.0 \%, P_{\mathrm{H}}=0.201 ; P_{\mathrm{z}}=0.015$; Fig. $3 c)$, whereas no significant association was found between Rac1 positive expression and tumor differentiation (OR=1.42, 95\% CI: 0.75-2.66, $I^{2}=55.0 \%$, $P_{\mathrm{H}}=0.038 ; P_{\mathrm{Z}}=0.281 ;$ Fig. $\left.3 \mathrm{~d}\right)$

\section{Sensitivity analysis of Racl for OS and DFS}

In order to examine the stability of the pooled HRs in OS and DFS analysis, sensitivity analysis was carried out with the successive omission of each study. The leaving-one-out study revealed that no study altered the pooled results significantly (Fig. 4a and $b$ ).

\section{Publication bias}

Neither Begg's nor Egger's tests showed a significant publication bias in OS and DFS (OS: Begg's test $P=0.631$, Egger's test $P=0.514$; DFS: Begg's test $P=$ 1.000 , Egger's test $P=0.838)$. Begg's funnel plot is demonstrated in Fig. 5, and no apparent asymmetry was found.

\section{Discussion}

To the best of our knowledge, this is the first meta-analysis focused on the relation between Rac1 expression and tumor survival. Both 1571 patients' pooled data for OS analysis and 815 patients' pooled data for DFS analysis showed that high Rac1 expression level was significantly correlated with poor survival in cancer patients. The probability of death in cancer patients with positive Rac1 expression was more than twice of that in patients with negative Rac1 expression. No significant heterogeneity was found in the OS. However, due to a relatively small number of studies included, a significant heterogeneity was shown in pooled HR for DFS. Pooled data showed that elevated Raclexpression was significantly linked to the malignancy-related behaviors such as blood vessel invasion, lymph metastasis, and late TNM stage, supporting the conclusion that high Rac1 expression could predict a poor prognosis for cancer patients.

By regulating cell skeleton and modulating the activity of a plethora of signaling in the body, Rac1 regulates many processes such as cell proliferation, migration, apoptosis, inflammation, and angiogenesis both in physiological and pathological conditions [37]. The role of Rac1 is crucial in oncogenesis. Tumor invasion and metastasis are two major malignancyrelated behaviors of tumor and have a decisive role in patients' survival [38]. It has been well established that the cell-cell adhesion and polarity loss plays an important role in the early stage of tumor metastasis [39]. Rac1 regulates the polarity of epithelial cells by interconnected with polarity associated proteins including Par, Scrible and Crumbs complexes, and these complexes control the model of cell actin cytoskeleton to build and maintain cell polarity and cell-cell junctions [6]. A recent study showed high Rac1 expression led to polarity change in invasive ductal carcinoma of breast and was positively associated with lymph metastasis and poor prognosis [35].

EMT has been considered as another critical decisive factor regulating tumor invasion and metastasis [40]. Epithelial cells lose their organization and transform to motile and invasive counterparts, promoting the invasion and metastasis of cancer. Rac1 mediated multiple functions indispensable for EMT such as cytoskeletal remodeling, cell adhesion, and transcriptional modulation [41]. An included study revealed that down-regulation of Rac1 inhibited the proliferation and EMT capability in an ovarian epithelial cell line, and silencing Rac1 in xenograft tumor model presented a significant increase in 
E-cadherin and loss of vimentin [17].

Overexpression of Rac1 was also reported to be associated with up-regulated N-cadherin and Vimen- tin level and negatively linked to E-cadherin level in lung cancer. Meanwhile, high Racl level was correlated with tumor metastasis and late TNM stage [34].

Table 2. Subgroup analysis of pooled HR for Racl impact on OS and DFS

\begin{tabular}{|c|c|c|c|c|c|c|c|c|c|c|}
\hline & \multicolumn{5}{|l|}{ OS } & \multicolumn{5}{|l|}{ DFS } \\
\hline & $\begin{array}{l}\text { Number of study } \\
\text { (sample size) }\end{array}$ & HR $(95 \% \mathrm{CI})$ & $I^{2}(\%)$ & $P_{\mathrm{H}}$ & $P z$ & $\begin{array}{l}\text { Number of study } \\
\text { (sample size) }\end{array}$ & HR (95\%CI) & $I^{2}$ & $P_{\mathrm{H}}$ & $P z$ \\
\hline All studies & $12(1571)$ & $2.02(1.70-2.31)^{\mathrm{a}}$ & 18.2 & 0.265 & $<0.001$ & $6(815)$ & $2.64(1.74-4.09)^{\mathrm{b}}$ & 60.4 & 0.027 & $<0.001$ \\
\hline Age $\geqq 60 y$ & $6(821)$ & $1.47(1.07-2.03)^{\mathrm{a}}$ & 0.0 & 0.532 & 0.018 & $2(168)$ & $1.73(0.93-3.23)^{\mathrm{a}}$ & 39.5 & 0.198 & 0.084 \\
\hline Age $<60 y$ & $5(648)$ & $2.06(1.44-2.93)^{\mathrm{a}}$ & 35.6 & 0.212 & $<0.001$ & $2(312)$ & $2.95(1.82-4.82)^{\mathrm{a}}$ & 49.5 & 0.160 & $<0.001$ \\
\hline Asian group & $11(1495)$ & $2.04(1.71-2.42)^{\mathrm{a}}$ & 24.0 & 0.215 & $<0.001$ & $5(755)$ & $2.58(1.58-4.21)^{b}$ & 67.9 & 0.027 & $<0.001$ \\
\hline European group & 1 & $1.56(0.61,4.01)$ & - & - & - & $1(60)$ & $3.22(1.04-9.98)$ & - & - & - \\
\hline Gastric cancer & $4(386)$ & $1.83(1.27-2.63)^{a}$ & 0.0 & 0.466 & 0.001 & - & - & - & - & - \\
\hline Non-small lung cancer & $2(264)$ & $2.16(1.45-3.24)^{\mathrm{a}}$ & 0.0 & 0.843 & $<0.001$ & - & - & - & - & - \\
\hline Other cancers & $6(921)$ & $2.10(1.52-2.90)^{\mathrm{b}}$ & 52.1 & 0.063 & $<0.001$ & $6(815)$ & $2.64(1.71,-4.09)^{\mathrm{b}}$ & 60.4 & 0.027 & $<0.001$ \\
\hline IHC cut off $\geqq 33 \%$ & $4(613)$ & $1.73(1.12-2.65)^{\mathrm{b}}$ & 57.8 & 0.068 & $<0.001$ & - & - & - & - & - \\
\hline IHC cut off $<33 \%$ & $7(850)$ & $2.35(1.87-2.85)^{b}$ & 0.0 & 0.873 & $<0.001$ & $5(707)$ & $3.00(1.91-4.71)^{\mathrm{b}}$ & 56.6 & 0.056 & $<0.001$ \\
\hline Follow up $<40$ month & $6(756)$ & $2.01(1.58-2.55)^{\mathrm{b}}$ & 0.0 & 0.792 & $<0.001$ & $2(395)$ & $2.02(1.43-2.87)^{a}$ & 0.0 & 0.661 & $<0.001$ \\
\hline Follow up $\geqq 40$ month & $5(755)$ & $2.02(1.37-3.00)^{b}$ & 58.7 & 0.046 & $<0.001$ & $4(420)$ & $3.15(1.67-5.95)^{b}$ & 66.0 & 0.032 & $<0.001$ \\
\hline Sample size $\geqq 100$ & $8(1257)$ & $1.94(1.61-2.34)^{\mathrm{a}}$ & 35.8 & 0.143 & $<0.001$ & $5(755)$ & $2.58(1.58-4.21)^{b}$ & 67.9 & 0.014 & $<0.001$ \\
\hline Sample size $<100$ & $4(314)$ & $2.40(1.63-3.53)^{a}$ & 0.0 & 0.657 & $<0.001$ & $1(60)$ & $3.22(1.04-9.98)$ & - & - & - \\
\hline Multivariate analysis & $6(861)$ & $2.42(1.89-3.10)^{a}$ & 0.0 & 0.871 & $<0.001$ & $4(432)$ & $2.58(1.19-5.56)^{b}$ & 69.8 & 0.019 & 0.005 \\
\hline Univariate analysis & $6(750)$ & $1.72(1.37-2.17)^{\mathrm{a}}$ & 34.8 & 0.176 & $<0.001$ & $2(383)$ & $2.65(1.55-4.53)^{\mathrm{b}}$ & 61.1 & 0.109 & $<0.001$ \\
\hline PT: no & 10 (1237) & $2.21(1.84-2.66)^{a}$ & 0.0 & 0.743 & $<0.001$ & $5(653)$ & $2.83(1.73-4.61)^{\mathrm{b}}$ & 66.0 & 0.019 & $<0.001$ \\
\hline PT: NA & $2(334)$ & $1.29(0.85-1.94)^{a}$ & 49.6 & 0.159 & 0.233 & $1(162)$ & $1.67(0.66-4.22)^{\mathrm{b}}$ & - & - & - \\
\hline
\end{tabular}

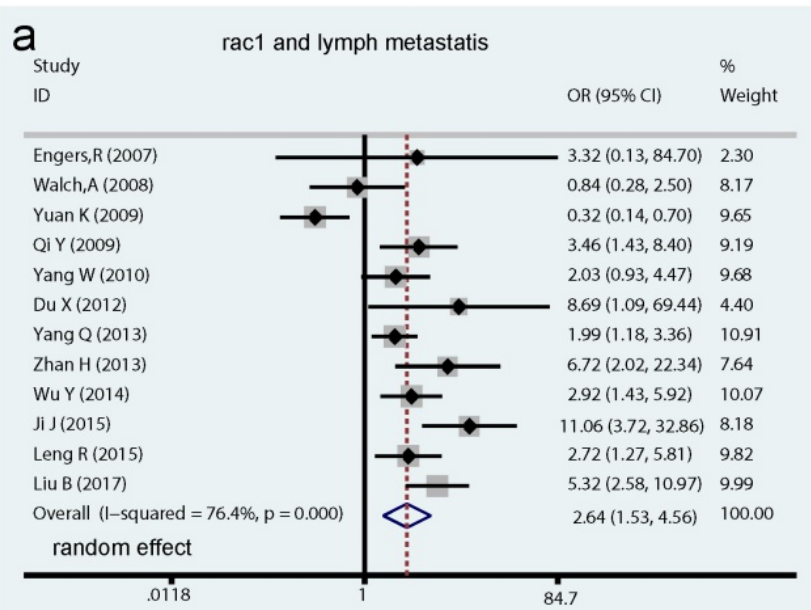

C Study rac1 and Blood vessel invasion

ID

(ng,

Engers, R (2007
Yang W (2010)

Yang W (2010)

DuX (2012)

$\mathrm{JiJ}(2015)$

Zhou Y (2016)

Overall (I-squared $=33.0 \%, \mathrm{p}=0.201)$

fixed effect .0092 b Study rac1 and Tumor stage/grade ID Engers, R (2007) Walch,A (2008) Yuan K (2009) Qi $Y$ (2009) Yang W (2010) DuX (2012) Yang Q (2013) Zhan H (2013) Wu Y (2014) Ji J (2015) Leng R (2015) Zhou Y (2016) Liu B (2017) Overall (I-squared $=69.7 \%, \mathrm{p}=0.000$ ) \begin{tabular}{c|c} 
random effect & \\
\hline 0264 & \\
\hline
\end{tabular} \begin{tabular}{c|c} 
random effect & : \\
\hline .0264 & 37.8
\end{tabular} OR $(95 \% \mathrm{Cl}) \quad$ Weight $2.28(0.79,6.61) \quad 7.05$ $0.64(0.21,1.92) \quad 6.84$ $2.31(1.04,5.11) \quad 8.53$ $3.16(1.29,7.75) \quad 7.95$ $8.98(3.04,26.51) \quad 6.95$ $5.60(1.19,26.31) \quad 4.90$ $2.10(1.24,3.54) \quad 10.04$ $6.72(2.02,22.34) \quad 6.36$ $5.13(2.49,10.55) \quad 8.94$ $2.69(1.05,6.90) \quad 7.69$ $16.54(7.23,37.83) 8.34$ $1.59(0.78,3.25) \quad 8.99$ $1.54(0.57,4.17) \quad 7.42$ $3.17(2.02,4.97) \quad 100.00$

d Study ID rac1 and tumor differetiation R $(95 \%$ Cl) Weight

Fig 3. Forrest plots of pooled odds ratios estimate for Racl expression and clinicopathological features. (a) pooled OR for Racl expression and cancer lymph metastasis. (b) pooled OR for Racl expression and tumor stage/grade. (c) pooled OR for Racl expression and blood vessel invasion. (d) pooled OR for Racl expression and tumor differentiation. 

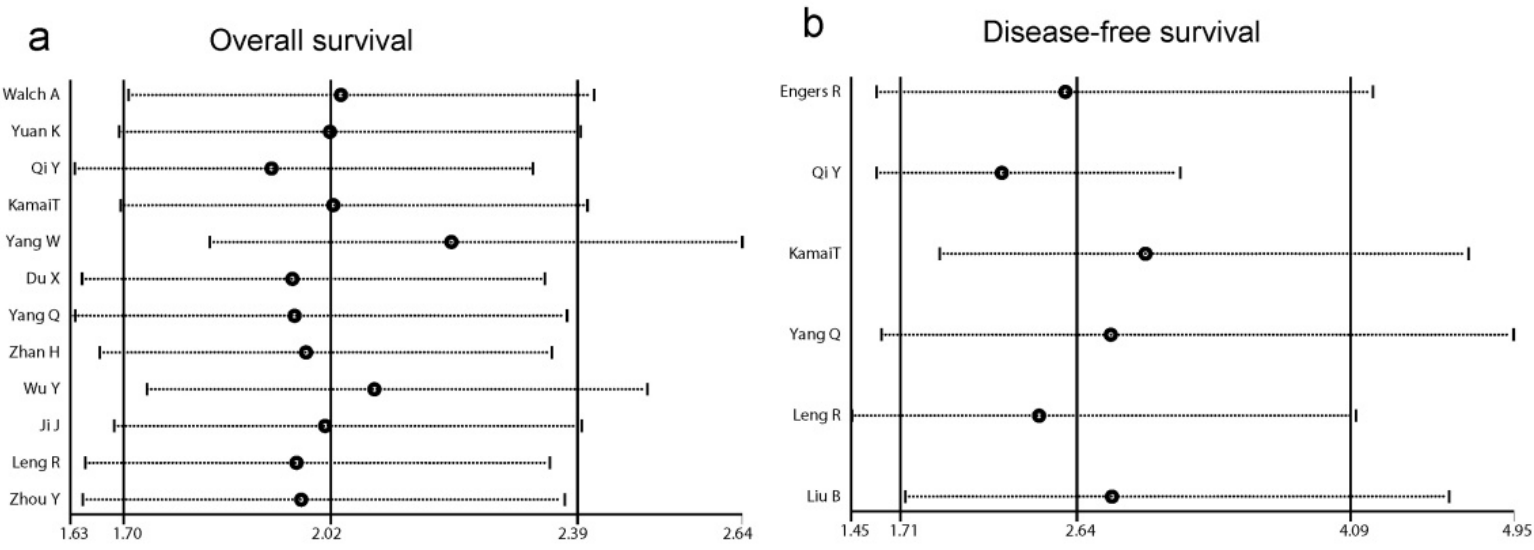

Fig 4. Forests plots of sensitivity analysis. (a) sensitivity analysis of overall survival (OS). (b) sensitivity analysis of disease-free survival (DFS).
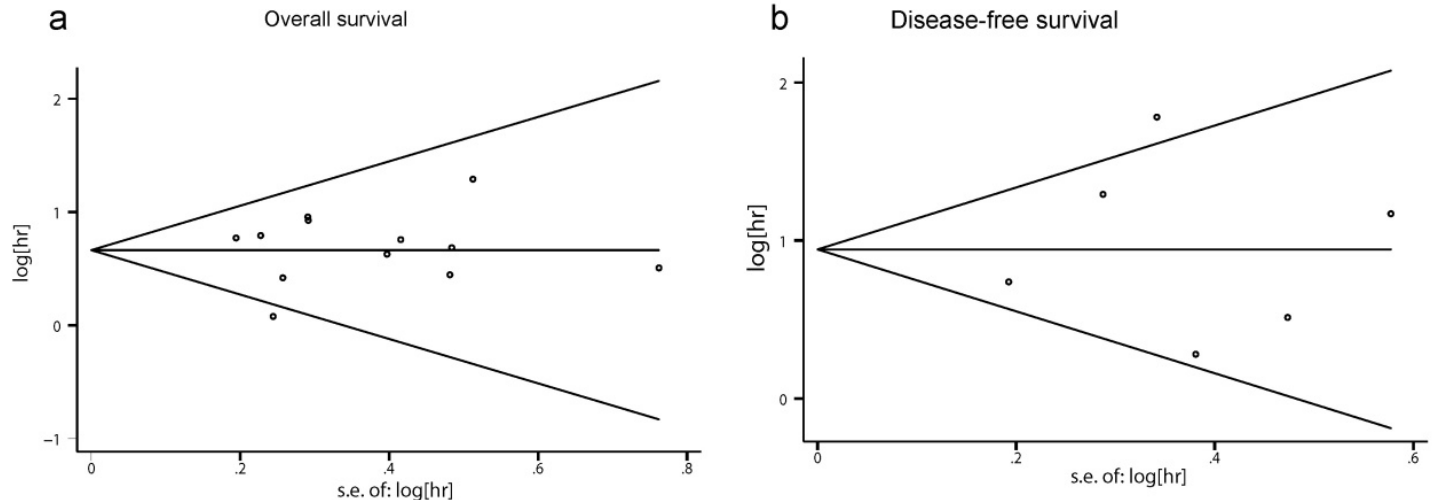

Fig 5. Begg's funnel plots for publication bias in Racl impact on the overall survival (OS) and disease-free survival (DFS). (a) Begg's funnel plot for OS analysis. (b) Begg's funnel plot for DFS analysis.

Our pooled data are special strong with the role of Rac1 in cancer invasive and metastasis behaviors. Early studies showed that treatment with NSC23766, a chemical inhibitor of Rac1, blocked the invasion and metastasis phenotype in multiple cancer cells [42]. It has also been shown that inhibition of Rac1 signaling in a murine lymphoma model abrogated tumor progression and metastasis in vivo [43]. This evidence demonstrated that Rac1 could be regarded as a therapeutic target for anticancer drugs.

In addition to the role of potential therapeutic target, Rac1 seems to be a good biomarker for monitoring the stage of tumor progression. It would be helpful to screen the biological features and provide appropriated treatment advises for cancer patients. Further studies are needed to reach an agreement on standard test method and cut-off values. A plethora of biomarkers of interaction was found in the microenvironment of tumor cells, thus Rac1 combination of other biomarkers, instead of Rac1 alone, would be beneficial to improve the clinical stage [44].

In spite of our efforts to reach an accurate and comprehensive meta-analysis, there are still some limitations in the present study. First, only eight of the fourteen included literature presented direct HRs, the
HRs of other studies were extracted from survival curves. This method of data extraction is prone to generate bias. We used the Engauge Digitizer software to extract data and avoid the possible bias by selecting appropriate time intervals. Secondly, the positive expression of Rac1 in most studies was detected using immune-histochemical method, which is a semi-quantitative method. Potential bias also could be generated due to the various definition of cut-off point of each study using IHC. Third, most of the studies included in this meta-analysis were performed in Asia, especially in China, and only two studies were conducted in Europe. More large-scale studies conducted among different countries and ethnicities are warranted to further investigate the prognostic value of Rac1 in cancer patients. Finally, a potential language bias may result from the included studies only being searched in English databases.

In summary, this study indicated that positive Rac1 expression predicted a poor prognosis of cancer patients. The conclusion was further supported by the correlation of Rac1 with progressive clinicopathological features, indicating Rac1 may serve as a potential molecular marker for monitoring cancer development. 


\section{Abbreviations}

CI: confidence interval; DFS: disease-free survival; EPC: esophageal cancer; HPC: hepatocellular cancer; HR: hazard ratio; IHC: immunohistochemistry; M: multivariate; NA: not available; NPC: nasopharyngeal cancer; NSCLC: non-small cell lung cancer; OS: overall survival; $P_{\mathrm{H}}: \quad P$ value for heterogeneity; PT: preoperative treatment; $P_{\mathrm{Z}}$ : $P$ value for $\mathrm{Z}$ test; $\mathrm{U}$ : univariate; WB: western blotting.

\section{Supplementary Material}

Supplementary table.

http://www.jcancer.org/v09p2571s1.pdf

\section{Acknowledgments}

This work was supported by the National Natural Science Foundation of China (81771029), Natural Science Foundation of Jiangsu Province (BK20150048), and Project Funded by the Priority Academic Program Development of Jiangsu Higher Education Institutions (2014-037).

\section{Competing Interests}

The authors have declared that no competing interest exists.

\section{References}

1. Torre LA, Bray F, Siegel RL, et al. Global cancer statistics, 2012. CA: a cancer journal for clinicians. 2015; 65: 87-108.

2. Kokka F, Bryant A, Brockbank E, et al. Hysterectomy with radiotherapy or chemotherapy or both for women with locally advanced cervical cancer. The Cochrane database of systematic reviews. 2015: CD010260.

3. Ding N, Pang Z, Shen H, et al. The Prognostic Value of PLR in Lung Cancer, a Meta-analysis Based on Results from a Large Consecutive Cohort. Sci Rep. 2016; 6: 34823

4. Yao GL, Fan YG, Zhai JM, et al. Radical gastrectomy with hepatoarterial catheter implantation for late-stage gastric cancer. World journal of gastroenterology. 2015; 21: 2754-8.

5. Ballard-Barbash R, Friedenreich CM, Courneya KS, et al. Physical activity, biomarkers, and disease outcomes in cancer survivors: a systematic review. Journal of the National Cancer Institute. 2012; 104: 815-40.

6. Bid HK, Roberts RD, Manchanda PK, et al. RAC1: An Emerging Therapeutic Option for Targeting Cancer Angiogenesis and Metastasis. Molecular Cancer Therapeutics. 2013; 12: 1925-34.

7. Li H, Peyrollier K, Kilic G, et al. Rho GTPases and cancer. BioFactors. 2014; 40: 226-35.

8. Mack NA, Georgiou M. The interdependence of the Rho GTPases and apicobasal cell polarity. Small GTPases. 2014; 5: 10.

9. Kamai T, Shirataki H, Nakanishi K, et al. Increased Rac1 activity and Pak1 overexpression are associated with lymphovascular invasion and lymph node metastasis of upper urinary tract cancer. BMC cancer. 2010; 10: 164

10. Cuadrado A, Martin-Moldes Z, Ye J, et al. Transcription factors NRF2 and NF-kappaB are coordinated effectors of the Rho family, GTP-binding protein RAC1 during inflammation. J Biol Chem. 2014; 289: 15244-58.

11. Kogai T, Liu YY, Mody K, et al. Regulation of sodium iodide symporter gene expression by Rac1/p38beta mitogen-activated protein kinase signaling pathway in MCF-7 breast cancer cells. J Biol Chem. 2012; 287: 3292-300.

12. Salker MS, Schierbaum N, Alowayed N, et al. LeftyA decreases Actin Polymerization and Stiffness in Human Endometrial Cancer Cells. Sci Rep. 2016; 6: 29370
13. Li A, Ma Y, Yu X, et al. Rac1 drives melanoblast organization during mouse development by orchestrating pseudopod- driven motility and cell-cycle progression. Dev Cell. 2011; 21: 722-34.

14. Koh W, Mahan RD, Davis GE. Cdc42- and Rac1-mediated endothelial lumen formation requires Pak2, Pak4 and Par3, and PKC-dependent signaling. Journal of cell science. 2008; 121: 989-1001.

15. D'Amico G, Jones DT, Nye E, et al. Regulation of lymphatic-blood vessel separation by endothelial Rac1. Development. 2009; 136: 4043-53.

16. Zhan H, Liang H, Liu X, et al. Expression of Rac1, HIF-1alpha, and VEGF in gastric carcinoma: correlation with angiogenesis and prognosis. Onkologie. 2013; 36: 102-7.

17. Leng $R$, Liao $G$, Wang $H$, et al. Rac1 expression in epithelial ovarian cancer: effect on cell EMT and clinical outcome. Medical oncology. 2015; 32: 329 .

18. Revach OY, Winograd-Katz SE, Samuels $Y$, et al. The involvement of mutant Rac1 in the formation of invadopodia in cultured melanoma cells. Exp Cell Res. 2016; 343: 82-8.

19. Santibanez JF, Kocic J, Fabra A, et al. Rac1 modulates TGF-beta1-mediated epithelial cell plasticity and MMP9 production in transformed keratinocytes. FEBS letters. 2010; 584: 2305-10.

20. Engers R, Ziegler S, Mueller M, et al. Prognostic relevance of increased Rac GTPase expression in prostate carcinomas. Endocrine-related cancer. 2007; 14: 245-56.

21. Walch A, Seidl S, Hermannstadter C, et al. Combined analysis of Rac1, IQGAP1, Tiam1 and E-cadherin expression in gastric cancer. Modern pathology : an official journal of the United States and Canadian Academy of Pathology, Inc. 2008; 21: 544-52.

22. Kai Yuan CQ, Ruheng Zheng. Prognostic signifi cance of immunohistochemical Rac1 expression in survival in early operable non-small cell lung cancer. Med Sci Monit. 2009; 15: 313-9.

23. Qi Y, Huang B, Yu L, et al. Prognostic value of Tiam1 and Rac1 overexpression in nasopharyngeal carcinoma. ORL; journal for oto-rhino-laryngology and its related specialties. 2009; 71: 163-71.

24. Yang W, Lv S, Liu X, et al. Up-regulation of Tiam1 and Rac1 correlates with poor prognosis in hepatocellular carcinoma. Japanese journal of clinical oncology. 2010; 40: 1053-9.

25. Chang $\mathrm{MH}$, Lee $\mathrm{K}$, Lee $\mathrm{KY}$, et al. Prognostic role of integrin beta1, E-cadherin, and rac1 expression in small cell lung cancer. APMIS : acta pathologica, microbiologica, et immunologica Scandinavica. 2012; 120: 28-38.

26. Du X, Wang S, Lu J, et al. Clinical value of Tiam1-Rac1 signaling in primary gallbladder carcinoma. Medical oncology. 2012; 29: 1873-8.

27. Silva AL, Carmo F, Bugalho MJ. RAC1b overexpression in papillary thyroid carcinoma: a role to unravel. European journal of endocrinology. 2013; 168: 795-804.

28. Yang Q, Luo GY, Li Y, et al. Expression of Rac-1 related to tumor depth, lymph node metastasis and patient prognosis in esophageal squamous cell carcinoma. Medical oncology. 2013; 30: 689.

29. Zhang JY, Zhang D, Wang EH. Overexpression of small GTPases directly correlates with expression of delta-catenin and their coexpression predicts a poor clinical outcome in nonsmall cell lung cancer. Molecular carcinogenesis. 2013; 52: 338-47.

30. Alonso-Espinaco V, Cuatrecasas $\mathrm{M}$, Alonso $\mathrm{V}$, et al. RAC1b overexpression correlates with poor prognosis in KRAS/BRAF WT metastatic colorectal cancer patients treated with first-line FOLFOX/XELOX chemotherapy. European journal of cancer. 2014; 50: 1973-81.

31. Wu YJ, Tang Y, Li ZF, et al. Expression and significance of Rac1, Pak1 and Rock1 in gastric carcinoma. Asia-Pacific journal of clinical oncology. 2014; 10: e33-9.

32. Ji J, Feng X, Shi M, et al. Rac1 is correlated with aggressiveness and a potential therapeutic target for gastric cancer. International journal of oncology. 2015; 46: 1343-53

33. Faria M, Capinha L, Simoes-Pereira J, et al. Extending the Impact of RAC1b Overexpression to Follicular Thyroid Carcinomas. International journal of endocrinology. 2016; 2016: 1972367.

34. Zhou Y, Liao Q, Han Y, et al. Rac1 overexpression is correlated with epithelial mesenchymal transition and predicts poor prognosis in non-small cell lung cancer. Journal of Cancer. 2016; 7: 2100-9.

35. Liu B, Xiong J, Liu G, et al. High expression of Rac1 is correlated with partial reversed cell polarity and poor prognosis in invasive ductal carcinoma of the breast. Tumour biology : the journal of the International Society for Oncodevelopmental Biology and Medicine. 2017; 39: 1010428317710908

36. Lo CK, Mertz D, Loeb M. Newcastle-Ottawa Scale: comparing reviewers' to authors' assessments. BMC Medical Research Methodology. 2014: 14:45. 
37. Marinkovic G, Heemskerk N, van Buul JD, et al. The Ins and Outs of Small GTPase Rac1 in the Vasculature. The Journal of pharmacology and experimental therapeutics. 2015; 354: 91-102.

38. Hou XL, Ji CD, Tang J, et al. FPR2 promotes invasion and metastasis of gastric cancer cells and predicts the prognosis of patients. Sci Rep. 2017; 7: 3153 .

39. Coradini D, Casarsa C, Oriana S. Epithelial cell polarity and tumorigenesis: new perspectives for cancer detection and treatment. Acta pharmacologica Sinica. 2011; 32: 552-64.

40. Diepenbruck M, Christofori G. Epithelial-mesenchymal transition (EMT) and metastasis: yes, no, maybe? Current opinion in cell biology. 2016; 43: 7-13.

41. Bendris N, Arsic N, Lemmers B, et al. Cyclin A2, Rho GTPases and EMT. Small GTPases. 2012; 3: 225-8.

42. Dokmanovic M, Hirsch DS, Shen $\mathrm{Y}$, et al. Rac1 contributes to trastuzumab resistance of breast cancer cells: Rac1 as a potential therapeutic target for the treatment of trastuzumab-resistant breast cancer. Mol Cancer Ther. 2009; 8: 1557-69.

43. Choudhari R, Minero VG, Menotti $M$, et al. Redundant and nonredundant roles for Cdc42 and Rac1 in lymphomas developed in NPM-ALK transgenic mice. Blood 2016; 127: 1297-306.

44. Schumacher TN, Kesmir C, van Buuren MM. Biomarkers in cancer immunotherapy. Cancer cell. 2015; 27: 12-4. 